\title{
STELLAR DYNAMICS OF LOW MASS STARS FROM THE SURFACE TO THE INTERIOR MEASURED BY COROT AND KEPLER
}

\author{
R.A. García ${ }^{1,2}$
}

\begin{abstract}
Continuous high-precision photometry of stars provided by space missions such as CoRoT, Kepler, and K2 represents a unique way to study stellar rotation and magnetism. The coupling of these studies of the surface dynamics with asteroseismology is changing our view to surface and internal dynamics. In this proceedings I will provide the latest developments in the understanding of surface and internal rotation and magnetic fields. I will also discuss the possible discovery of strong internal magnetic fields of dynamo origin in the convective cores of stars above 1.2-1.4 solar masses. I will finish by providing constraints on gyrochronology laws for low-mass stars and put the Sun into context of its magnetism when compared to other solar-analog stars.
\end{abstract}

\section{Introduction}

Current photometric observations obtained from space missions such as CoRoT (Baglin et al. 2006), Kepler (Borucki et al. 2010), and K2 (Howell et al. 2014) allow us to monitor stellar brightness in a continuous way with very high precision for hundred of thousand stars (e.g. Chaintreuil et al. 2016; Mathur et al. 2017). These long time series, typically from 1 month to several years, have revolutionized the study of surface stellar dynamics. For stars exhibiting magnetic features on their surfaces (e.g. starspots), the stellar brightness is modulated with a period proportional to the stellar rotation rate at the latitude in which these spots are located (e.g. García et al. 2009, 2012, 2014a; Nielsen et al. 2013; McQuillan et al. 2013b; 2014; Ceillier et al. 2016; Beck et al. 2017; Ceillier et al. 2017). Therefore, in these stars, it is possible to measure the average surface rotation rate as a function of time. Moreover, if the star has a surface differential rotation

\footnotetext{
${ }^{1}$ IRFU, CEA, Université Paris-Saclay, 91191 Gif-sur-Yvette, France

${ }^{2}$ Université Paris Diderot, AIM, Sorbonne Paris Cité, CEA, CNRS, 91191 Gif-sur-Yvette, France
} 
and there are several spots evolving at different latitudes, it is possible to measure a lower limit of this differential rotation (e.g. Fröhlich et al. 2012; Reinhold \& Reiners 2013; Lanza et al. 2014; Reinhold \& Gizon 2015; Santos et al. 2017).

If there is a magnetic cycle in a star (regular or not), the size and number of spots at the surface changes inducing a long term evolution of the stellar surface brightness that can be used as a proxy of the surface magnetism (e.g. Oláh et al. 2009; Basri et al. 2013; Mathur et al. 2013; Salabert et al. 2017). To ensure that the brightness variations are related to the magnetic evolution and not to other sources such as convection or pulsations, it has been proposed to sample such variations in chunks of three to five times the average stellar rotation rate (Mathur et al. 2014b). The corresponding magnetic proxy has been called photospheric $\mathrm{S}$ index, $S_{p h}$, in analogy to the Chromospheric S index (Wilson 1978, Noyes et al. 1984a) and has been used to study stellar magnetism of different type of stars from F to M dwarfs (e.g. Mathur et al. 2014a, 2014b; Vida et al. 2014; Salabert et al. 2016a, 2016b).

Long photometric space observations have also enabled the asteroseismic revolution. Asteroseismology has provided global stellar parameters such as masses and radii for thousands of stars from the direct characterization of the seismic properties (e.g. Chaplin et al. 2014; Serenelli et al. 2017; Silva Aguirre et al. 2017 ), or by indirect methods from the study of the convective properties (Mathur et al. 2011b; Kallinger et al. 2014) or the study of the variability (Bastien et al. 2013, 2016; Bugnet et al. 2017, 2018). Moreover, today it is possible to probe stellar interiors and constrain their structures (e.g. Mathur et al. 2010; Metcalfe et al. 2010; Mathur et al. 2011a; Deheuvels \& Michel 2011; Mathur et al. 2012; Metcalfe et al. 2012, 2014; Creevey et al. 2017; Silva Aguirre et al. 2017) and dynamic properties (e.g. Deheuvels et al. 2012, 2014; Mosser et al. 2012b; Gizon et al. 2013; García et al. 2014c; Benomar et al. 2015; Nielsen et al. 2015, 2017; Di Mauro et al. 2016) for hundreds of main-sequence dwarfs and tens of thousands of red giants. The temporal variation of seismic parameters have also allowed us to unveil the presence of magnetic activity cycles in several main-sequence dwarfs (e.g. García et al. 2010; Mathur et al. 2013; Régulo et al. 2016; Salabert et al. 2016b; Kiefer et al. 2017; Santos et al. 2018).

In this proceedings the latest results concerning surface and internal rotation and magnetism combining photometric variations and asteroseismology are reviewed. Special attention has been given to update the bibliography to the moment in which this proceedings has been written.

\section{Stellar rotation}

\subsection{Surface}

Using Kepler data, Nielsen et al. (2013), Reinhold et al. (2013), and McQuillan et al. (2014) have determined surface rotations, $P_{\text {rot }}$, of tens of thousands of stars. All of them used Simple Aperture Photometry (SAP) time series, calibrated using PDC (Pre-search Data Conditioning) algorithms (Stumpe et al. 2012; 
Smith et al. 2012; Stumpe et al. 2014). Originally developed to maximize the exoplanet detections, PDC could filter stellar periods longer than 3 days (see for example the discussion in García et al. 2013). Therefore, it is recommended to use other calibration procedures ensuring that the low-frequency stellar signals including rotation are not filtered out. This is the objective of the KADACS light curves (García et al. 2011), which also include an effective interpolation procedure based on in-painting techniques (Pires et al. 2015) to reduce the effects of the regular gaps in the Kepler light curves (García et al. 2014b), or the newly developed methods based on gaussian processes (Aigrain et al. 2017).

Surface rotation can be extracted by selecting the highest peak in the lowfrequency part of the power spectrum (e.g. Barban et al. 2009; Campante et al. 2011; Nielsen et al. 2013). When a star has two spots at approximately $180^{\circ}$, the highest peak is the second harmonic and the retrieved peak is half of the average $P_{\text {rot }}$ (see an extensive discussion in García et al. 2013). To minimize this problem, it is recommended to study the rotation in the time domain through the use of autocorrelation functions (e.g. McQuillan et al. 2013a). This methodology is more robust and it is less sensitive to the above mentioned effect of measuring half rotation periods. Finally, it is possible to study rotation in a time-period diagram based on wavelet transforms (e.g. García, et al. 2009; Mathur et al. 2010). This method has the advantage of highlighting possible instrumental problems in a given quarter. In a benchmark comparaison of different groups and techniques, Aigrain et al. (2015) showed that the best combination of completeness and reliability $P_{\text {rot }}$ were obtained by combining different detrending algorithms and several periodsearch methods.

\subsection{Calibrating gyrochronology: Contribution of asteroseismology}

One of the fundamental properties of stars is their ages. But ages are not easy to determine and the classical isochrone method (Sandage 1962; Demarque \& Larson 1964 ) is not precise enough to impose strict constraints to modern astrophysical problems, for example, to accurately date extrasolar systems of field stars. With the discovery of the evolution of chromospheric activity in cool stars (Wilson 1978; Skumanich 1972; Noyes et al. 1984b) activity-age relations developed. However, chromospheric activity can change in short time scales due to activity cycles, rotation phases, etc, limiting the precision in the age determination to around $50 \%$ (see for example Barnes 2007). Thus, rotation-age relations were proposed by Skumanich (1972) using averaged $v \sin (i)$ and known ages, leading to gyrochronology, i.e. the determination of the age from the measurement of the stellar rotation period in cool main-sequence dwarfs. Having an external convective region, these stars lose angular momentum with time through stellar winds. The measure of the rotation rate is then an indication of their ages. The use of the rotation period instead of the projected rotation velocity allows one to avoid the dependency on the stellar inclination angle.

To properly calibrate gyrochronology, stars belonging to young and intermediateage clusters $(<2.5 \mathrm{Gyr})$ and the Sun are commonly used (see for example 
Meibom et al. 2015). Thanks to space photometry, this has been extended to old open clusters such as M67 (Barnes et al. 2016). However, these rotation periods need to be taken with caution as "the sensibility drops rapidly with increasing period and decreasing amplitude, maxing at $15 \%$ recovery rate for the solar case (i.e. 25 d period, 0.1\% amplitude)" (as discussed by Esselstein et al. 2018). Another interesting calibration set for old field stars is the seismic sample for which stellar ages are accurately computed through asteroseismic techniques (García et al. 2014a; Angus et al. 2015; Davies et al. 2015). Field stars of the same mass or above the Sun have an anomalous fast rotation compared to the general predictions of the gyrochronology laws. Although still debated, van Saders et al. (2016) explained these discrepancy by a weakened magnetic braking. This result might suggest a fundamental change in the nature of evolved stellar dynamos, with the Sun being close to the critical transition to much weaker magnetized winds that occurs to all middle-aged stars (Metcalfe \& van Saders 2017). If confirmed, the diagnostic power of gyrochronology would be limited to stars younger than halfway their main-sequence lifetimes.

\subsection{Internal}

One of the main successes of helioseismology has been the inference of the solar internal differential rotation (e.g. Thompson et al. 1996; Chaplin et al. 1999; Thompson et al. 2003; García 2004) including hints of the inner average core spin rate (García et al. 2007; Fossat et al. 2017). When extending this analysis to other stars, the precision and the extension of the region to be explored depends on our ability to probe the inner regions, through mixed modes (Beck et al. 2011) in subgiants and red giants, or only the external regions, in main-sequence stars.

To have a general overview of the internal rotation rate of solar-like stars through evolution we start by main-sequence solar-like dwarfs. In these stars, neither mixed modes nor g modes have been measured. The only stratified information that can be obtained is through the careful analysis of acoustic modes. Unfortunately, although low-degree p modes penetrate deep in the stellar interior, they spend a small fraction of their time in the deep interior and the amount of information they can provide from these regions is small.

Benomar et al. (2015) analyzed CoRoT and Kepler cool dwarfs and computed rotation splittings for $\ell=1$ and 2 modes and the stellar inclination angle. Assuming that the stellar interior is composed of a radiative and a convective region rotating uniformily and using surface rotation rates deduced from spectroscopy or directly extracted from the Kepler photometry (García et al. 2014a) as a good indication of the average rotation rate of the external convective region (as in the solar case, see also the discussion in Davies et al. 2015, for the 16 Cyg. system), they obtained an estimation of the rotation rate in the internal radiative region. They found that for all the stars in the sample but one, the gradient between the two zones is less than 2, which implies a rather flat equatorial rotation rate similar to the equatorial solar rotation profile (see for example Fig. 9 in García et al. 2008). Moreover, because the internal structure of similar 
solar-like stars is comparable, a large number of those comparable stars could be use simultaneously to constrain their average radial differential rotation gradient in an ensemble way (Schunker et al. 2016).

Leaving the main sequence (Deheuvels et al. 2012, 2014), studied the internal rotation in subgiants and young red giants. To do so, they first characterized the rotational splittings of mixed modes in KIC 7341231. A clear gradient where found with larger splittings for modes with a larger g-mode contribution. This implied a faster core rotation when compared with the external regions. This analysis was then extended to 6 more stars (Deheuvels et al. 2014) obtaining similar results with a faster rotation rate in the core than in the surface. When using $\log g$ as a proxy of age, the core is contracting, the internal rotation is smoothly spinning up while the surface rotation is gradually slowing down as a consequence of the expansion of the external layers. This result is predicted by theory.

In the red giant phase, the first measurements of the gradient in RGB stars showed larger values than in main sequence or during the subgiant phase (e.g. Beck et al. 2012; Di Mauro et al. 2016). However, the spinning rate is too low when compared with theoretical expectations (e.g. Ceillier 2013) and a revision of angular momentum transport mechanisms is required (see discussions in, e.g. Marques et al. 2013; Mathis 2013; Tayar \& Pinsonneault 2013; Eggenberger et al. 2017). Moreover, the evolution of the core rotation rate is different to what it was expected from theory. Thus, Mosser et al. (2012b) showed that the core rotation spins down when stars evolve in the RGB, i.e. when the radius increase for a given stellar mass. When stars reach the clump, the core rotation is reduced again. This can be partially explained by the expansion of the non-degenerate helium burning core (Iben 1971; Sills \& Pinsonneault 2000), but it is required a significant transfer of internal angular momentum from the inner to the outer layers in this evolutionary phase. A detailed analysis of seven second clump stars revealed a core-to-envelope gradient in a range 1.8 to 3.2 (Deheuvels et al. 2015). However, the latest results of the core-rotation rates by (Gehan et al. 2018) suggest that instead of a slight spin down of the core during the RGB phase, the core rotation seems to be constant independently of the mass.

\section{Stellar magnetism}

\subsection{Surface}

Solar-like pulsating stars are characterized by having an external convective zone that excites oscillation modes. Convection and rotation usually generate dynamo magnetic fields in the surface of these stars that can then be measured and characterized.

Using directly the photometric time series obtained from CoRoT and Kepler, it is possible to obtain indications of the variability of the stars at different time scales (Basri et al. 2013). To ensure a magnetic origin of this variability, Mathur et al. (2014b) demonstrated that by taking chunks of 3-5 times the rotation period, the average resultant variability is a good proxy of the surface magnetism. 
This $S_{\mathrm{ph}}$ photometric activity proxy was recently applied to the Sun (Salabert et al. 2017) using Sun-as-a-star VIRGO/SPM photometric light curves as well as Doppler velocity GOLF time series (García et al. 2005). The comparison with different solar magnetic proxies showed hysteresis patterns between all the proxies (e.g. Bachmann \& White 1994; Jimenez-Reyes et al. 1998; Tripathy et al. 2001). The hysteresis effects are a consequence of the different response to the magnetic perturbations at different heights of the solar atmosphere, which could also experience a temporal lag. This hysteresis pattern between chromospheric and photospheric proxies is also visible in other stars such as the young solar analog KIC 10644253 (Salabert et al. 2016a).

Using an average $S_{\mathrm{ph}}$ for each solar-like pulsating dwarf observed by Kepler during the survey phase (Chaplin et al. 2014; García et al. 2014a) demonstrated that the surface magnetism of this ensemble set (Chaplin et al. 2011c) is comparable to the one of the Sun. A detailed analysis of the photospheric and chromospheric activity of eighteen solar-analogs confirmed this result (Salabert et al. 2016a).

Stars with larger surface magnetic variability show either weak or no oscillation modes (Mosser et al. 2009; Chaplin et al. 2011a), which has an impact on the prediction of stellar oscillations (Chaplin et al. 2011b). Moreover, the amplitude of the oscillation modes is anticorrelated with the magnetic activity cycle as it was already measured in the Sun and in the CoRoT target HD 49933 (García et al. 2010) and later in several Kepler stars (Kiefer et al. 2017). However, according to the analyses done by Santos et al. (2018), 20\% of their Kepler sample present a positive correlation between the temporal evolution of the mode heights and the frequency shifts.

\subsection{Internal}

While the variation of the averaged frequency shifts with time provide information about possible on-going magnetic activity cycles (e.g. Kiefer et al. 2017; Salabert et al. 2018; Santos et al. 2018), the frequency dependence of the p-mode frequency shifts can provide information about the structural changes occurring in stellar interiors with magnetic activity for solar-like dwarfs. In the case of the Sun, these changes are located in a thin layer right beneath the solar photosphere (e.g. Libbrecht \& Woodard 1990; Basu 2016). More precisely, Basu et al. (2012) suggested that in cycle 23 those changes were localized mainly in a region above about $0.996 R_{\odot}$. Using longer time series, Jain et al. (2018) have showed that structural and magnetic changes responsible for the frequency shifts remained comparable between the last two cycles (23 and 24), while they are different from what was found in cycle 22 .

This linear dependence of the frequency shifts with frequency was also found in other stars: first in the CoRoT target HD 49933 (Salabert et al. 2011) and later in the young solar analog KIC10644253 (Salabert et al. 2016b). In both cases, the perturbation in the acoustic modes was found to be located close 
to the photosphere. However, in four stars of the Kepler sample analyzed by Salabert et al. (2018), KIC 5184732, KIC 8006161, KIC 8379927, and KIC 11081729, the frequency shifts normalized by the mode inertia show an oscillatory behavior instead of a linear one. This suggests that the frequency perturbation could be produced in a thin layer inside the resonant cavity of the acoustic modes because in that case, this frequency dependence of the frequency shifts would follow a sinusoidal function. Before driving firm conclusions about the positions and mechanisms responsible for the frequency shifts in stars, a larger stellar sample is required. However, we can already say today that the picture seems to be much more complicated than what it was outlined from the unique analysis of the Sun.

The asteroseismic analysis of thousands of red giants has not yet reported the measure of any frequency shifts in the acoustic modes. This is probably due to a very small magnetic activity in the external layers of these stars. The same can be said about the study of mixed modes. Up to now, neither frequency shifts nor magnetic splittings have been reported.

Things changed in 2011, at the 4th Kepler Asteroseismic Science Consortium (KASC-4) meeting, when García et al. showed two red-giant stars observed by Kepler in which the amplitudes of the dipole modes were unexpectedly small. Then, Mosser et al. (2012a) realized that about $5 \%$ of their sample of 800 red giants had low-visibility dipolar modes. Fuller et al. (2015) outlined an explanation through the presence of a strong magnetic field in the core of these giants. They called this mechanism the "magnetic green house effect" because the magnetic field acts as a green house trapping the energy of the modes propagating in the core (see also Cantiello et al. 2016). Later, Stello et al. (2016) suggested that such magnetic field could be of dynamo origin because after analyzing 13,000 red giants there were no depressed dipolar modes for masses below 1.2 to $1.3 M_{\odot}$. In other words, only stars developing a convective core during their main-sequence life are affected, reaching about half of the stars for masses around $1.6 M_{\odot}$. Although this explanation could solve the problem of the origin of magnetic white dwarfs (which are the cores of these red giants), the result is still controversial. Indeed, these dipolar depressed modes were described as a normal mixed-mode pattern by Mosser et al. (2017), while the green house effect mechanism expects full suppression of the dipolar mixed modes. Only some power of the pure dipolar acoustic modes could be visible in the power spectrum (Fuller et al. 2015). Unfortunately, Mosser et al. (2017) did not provide any further explanation of the origin of the suppression of the dipolar mixed-mode power. Nearly at the same time, Loi \& Papaloizou (2017) presented another mechanism that damps the oscillations of stars harboring magnetized cores via resonant interactions with standing Alfvén modes of high harmonic index. With this mechanism, the magnetic field could still be responsible for the reduction in power of the dipolar modes while allowing to have some mixed-mode energy. As it is explained in this work: "The damping rates produced by this mechanism are quantitatively on par with those associated with turbulent convection, and in the range required to explain observations, for 
realistic stellar models and magnetic field strengths". The last word is not said yet in this topic and the community is actively studying these stars to better understand the mechanism responsible for the reduction in power of these dipolar modes.

\section{Conclusions}

The high-precision photometry of thousands of stars from space has put stellar dynamics in the center of many important studies. In the last few years there has been a lot of observational new discoveries allowing us to develop and refine theoretical models. This not only affects stellar physics but also the Sun and the stellar magnetic evolution that can change the way in which our star could be conceived in the future if it is transiting to a more quite magnetic phase as lately suggested.

K2 and TESS missions, as well as the future ESA M3 PLATO mission (Rauer et al. 2014) will provide a wealth of data. The future cannot be more promising for this field of research.

Funding for the Kepler Discovery mission is provided by National Aeronautics and Space Administrations's Science Mission Directorate. The authors wish to thank the entire Kepler team, without whom these results would not have been obtained. The CoRoT space mission has been developed and is operated by Centre National d'Etudes Spatiales (CNES), with contributions from Austria, Belgium, Brazil, the European Space Agency (RSSD and Science Program), Germany and Spain. The author thanks the French Programme National de Physique Stellaires for support as well as the CNES for supporting CoRoT and GOLF/SoHO activities at the DAp, CEA-Saclay. The author also acknowledges the support of the European Community's Seventh Framework Programme (FP7/2007-2013) under grant agreement No. 269194 (IRSES/- ASK) and No. 312844 (SPACEINN).

\section{References}

Aigrain, S., Llama, J., Ceillier, T., et al., 2015, MNRAS, 450, 3211

Aigrain, S., Parviainen, H., Roberts, S., Reece, S., \& Evans, T., 2017, MNRAS, 471, 759

Angus, R., Aigrain, S., Foreman-Mackey, D., \& McQuillan, A., 2015, MNRAS, 450, 1787

Bachmann, K.T., \& White, O.R., 1994, Sol. Phys., 150, 347

Baglin, A., Auvergne, M., Boisnard, L., et al., 2006, in COSPAR, Plenary Meeting, Vol. 36, 36th COSPAR Sci. Assembly, 3749

Barban, C., Deheuvels, S., Baudin, F., et al., 2009, A\&A, 506, 51

Barnes, S.A., 2007, ApJ, 669, 1167

Barnes, S.A., Weingrill, J., Fritzewski, D., Strassmeier, K.G., \& Platais, I., 2016, ApJ, 823,16

Basri, G., Walkowicz, L.M., \& Reiners, A., 2013, ApJ, 769, 37

Bastien, F.A., Stassun, K.G., Basri, G., \& Pepper, J., 2013, Nature, 500, 427

Bastien, F.A., Stassun, K.G., Basri, G., \& Pepper, J., 2016, ApJ, 818, 43 
Basu, S., 2016, Living Rev. Solar Phys., 13, 2

Basu, S., Broomhall, A.-M., Chaplin, W.J., \& Elsworth, Y., 2012, ApJ, 758, 43

Beck, P.G., Bedding, T.R., Mosser, B., et al., 2011, Science, 332, 205

Beck, P.G., do Nascimento, Jr., J.-D., Duarte, T., et al., 2017, A\&A, 602, A63

Beck, P.G., Montalban, J., Kallinger, T., et al., 2012, Nature, 481, 55

Benomar, O., Takata, M., Shibahashi, H., Ceillier, T., \& García, R.A., 2015, MNRAS, 452,2654

Borucki, W.J., Koch, D., Basri, G., et al., 2010, Science, 327, 977

Bugnet, L., Garcia, R.A., Davies, G.R., Mathur, S., \& Corsaro, E., 2017, ArXiv 1711.02890

Bugnet, L., Garcia, R.A., Davies, G.R., et al., 2018, A\&A, submitted

Campante, T.L., Handberg, R., Mathur, S., et al., 2011, A\&A, 534, A6

Cantiello, M., Fuller, J., \& Bildsten, L., 2016, ApJ, 824, 14

Ceillier, T., Eggenberger, P., García, R.A., \& Mathis, S., 2013, A\&A, 555, A54

Ceillier, T., Tayar, J., Mathur, S., et al., 2017, A\&A, 605, A111

Ceillier, T., van Saders, J., García, R.A., et al., 2016, MNRAS, 456, 119

Chaintreuil, S., Bellucci, A., Baudin, F., et al., 2016, II.5 Where to find the CoRoT data?, ed. CoRot Team, 109

Chaplin, W. J., Basu, S., Huber, D., et al., 2014, ApJS, 210, 1

Chaplin, W.J., Bedding, T.R., Bonanno, A., et al., 2011a, ApJ, 732, L5

Chaplin, W.J., Christensen-Dalsgaard, J., Elsworth, Y., et al., 1999, MNRAS, 308, 405

Chaplin, W.J., Kjeldsen, H., Bedding, T.R., et al., 2011b, ApJ, 732, 54

Chaplin, W.J., Kjeldsen, H., Christensen-Dalsgaard, J., et al., 2011c, Science, 332, 213

Creevey, O.L., Metcalfe, T.S., Schultheis, M., et al., 2017, A\&A, 601, A67

Davies, G.R., Chaplin, W.J., Farr, W.M., et al., 2015, MNRAS, 446, 2959

Deheuvels, S., Ballot, J., Beck, P.G., et al., 2015, A\&A, 580, A96

Deheuvels, S., Doğan, G., Goupil, M.J., et al., 2014, A\&A, 564, A27

Deheuvels, S., García, R.A., Chaplin, W.J., et al., 2012, ApJ, 756, 19

Deheuvels, S., \& Michel, E., 2011, A\&A, 535, A91

Demarque, P.R., \& Larson, R.B., 1964, ApJ, 140, 544

Di Mauro, M.P., Ventura, R., Cardini, D., et al., 2016, ApJ, 817, 65

Eggenberger, P., Lagarde, N., Miglio, A., et al., 2017, A\&A, 599, A18

Esselstein, R., Aigrain, S., Vanderburg, A., et al., 2018, ArXiv e-prints

Fossat, E., Boumier, P., Corbard, T., et al., 2017, A\&A, 604, A40

Fröhlich, H.-E., Frasca, A., Catanzaro, G., et al., 2012, A\&A, 543, A146

Fuller, J., Cantiello, M., Stello, D., Garcia, R.A., \& Bildsten, L., 2015, Science, 350, 423

García, R.A., Ceillier, T., Campante, T.L., et al., 2012, in Astronomical Society of the Pacific Conference Series, Vol. 462, Progress in Solar/Stellar Physics with Helio- and Asteroseismology, ed. H. Shibahashi, M. Takata, \& A.E. Lynas-Gray, 133

García, R.A., Ceillier, T., Mathur, S., \& Salabert, D., 2013, in Astronomical Society of the Pacific Conference Series, Vol. 479, Astronomical Society of the Pacific Conference Series, ed. H. Shibahashi \& A.E. Lynas-Gray, 129

García, R.A., Ceillier, T., Salabert, D., et al., 2014a, A\&A, 572, A34 
García, R.A., Corbard, T., Chaplin, W.J., et al., 2004, Sol. Phys., 220, 269

García, R.A., Hekker, S., Stello, D., et al., 2011, MNRAS, 414, L6

García, R.A., Mathur, S., Ballot, J., et al., 2008, Sol. Phys., 251, 119

García, R.A., Mathur, S., Pires, S., et al., 2014b, A\&A, 568, A10

García, R.A., Mathur, S., Salabert, D., et al., 2010, Science, 329, 1032

García, R.A., Pérez Hernández, F., Benomar, O., et al., 2014c, A\&A, 563, A84

García, R.A., Régulo, C., Samadi, R., et al., 2009, A\&A, 506, 41

García, R.A., Turck-Chièze, S., Boumier, P., et al., 2005, A\&A, 442, 385

García, R.A., Turck-Chièze, S., Jiménez-Reyes, S.J., et al., 2007, Science, 316, 1591

Gehan, C., Mosser, B., Michel, E., Samadi, R., \& Kallinger, T., 2018, ArXiv e-prints

Gizon, L., Ballot, J., Michel, E., et al., 2013, Proceedings of the Natl. Acad. Sci., 110, 13267

Howell, S.B., Sobeck, C., Haas, M., et al., 2014, PASP, 126, 398

Iben, Jr., I., 1971, PASP, 83, 697

Jain, K., Tripathy, S., Hill, F., et al., 2018, ArXiv e-prints

Jimenez-Reyes, S.J., Regulo, C., Palle, P.L., \& Roca Cortes, T., 1998, A\&A, 329, 1119

Kallinger, T., De Ridder, J., Hekker, S., et al., 2014, A\&A, 570, A41

Kiefer, R., Schad, A., Davies, G., \& Roth, M., 2017, A\&A, 598, A77

Lanza, A.F., Das Chagas, M.L., \& De Medeiros, J.R., 2014, A\&A, 564, A50

Libbrecht, K.G., \& Woodard, M.F., 1990, Nature, 345, 779

Loi, S.T., \& Papaloizou, J.C.B., 2017, MNRAS, 467, 3212

Marques, J.P., Goupil, M.J., Lebreton, Y., et al., 2013, A\&A, 549, A74

Mathis, S., 2013, in Lecture Notes in Physics Springer, Berlin, Verlag (ed. M. Goupil, K. Belkacem, C. Neiner, F. Lignières, \& J.J. Green), 865, 23

Mathur, S., García, R.A., Ballot, J., et al., 2014a, A\&A, 562, A124

Mathur, S., García, R.A., Catala, C., et al., 2010, A\&A, 518, A53

Mathur, S., García, R.A., Morgenthaler, A., et al., 2013, A\&A, 550, A32

Mathur, S., Handberg, R., Campante, T.L., et al., 2011a, ApJ, 733, 95

Mathur, S., Hekker, S., Trampedach, R., et al., 2011b, ApJ, 741, 119

Mathur, S., Huber, D., Batalha, N.M., et al., 2017, ApJS, 229, 30

Mathur, S., Metcalfe, T.S., Woitaszek, M., et al., 2012, ApJ, 749, 152

Mathur, S., Salabert, D., García, R.A., \& Ceillier, T., 2014b, J. Space Weather and Space Clim., 4, A15

McQuillan, A., Aigrain, S., \& Mazeh, T., 2013a, MNRAS, 432, 1203

McQuillan, A., Mazeh, T., \& Aigrain, S., 2013b, ApJ, 775, L11

McQuillan, A., Mazeh, T., \& Aigrain, S., 2014, ApJS, 211, 24

Meibom, S., Barnes, S.A., Platais, I., et al., 2015, Nature, 517, 589

Metcalfe, T.S., Chaplin, W.J., Appourchaux, T., et al., 2012, ApJ, 748, L10

Metcalfe, T.S., Creevey, O.L., Doğan, G., et al., 2014, ApJS, 214, 27

Metcalfe, T.S., Monteiro, M.J.P.F.G., Thompson, M.J., et al., 2010, ApJ, 723, 1583

Metcalfe, T.S., \& van Saders, J., 2017, Sol. Phys., 292, 126

Mosser, B., Belkacem, K., Pinçon, C., et al., 2017, A\&A, 598, A62 
Mosser, B., Elsworth, Y., Hekker, S., et al., 2012a, A\&A, 537, A30

Mosser, B., Goupil, M.J., Belkacem, K., et al., 2012b, A\&A, 548, A10

Mosser, B., Michel, E., Appourchaux, T., et al., 2009, A\&A, 506, 33

Nielsen, M. B., Gizon, L., Schunker, H., \& Karoff, C., 2013, A\&A, 557, L10

Nielsen, M.B., Schunker, H., Gizon, L., \& Ball, W.H., 2015, A\&A, 582, A10

Nielsen, M.B., Schunker, H., Gizon, L., Schou, J., \& Ball, W.H., 2017, A\&A, 603, A6

Noyes, R.W., Hartmann, L.W., Baliunas, S.L., Duncan, D.K., \& Vaughan, A.H., 1984a, ApJ, 279, 763

Noyes, R.W., Weiss, N.O., \& Vaughan, A.H., 1984b, ApJ, 287, 769

Oláh, K., Kolláth, Z., Granzer, T., et al., 2009, A\&A, 501, 703

Pires, S., Mathur, S., García, R.A., et al., 2015, A\&A, 574, A18

Rauer, H., Catala, C., Aerts, C., et al., 2014, Experimental Astronomy, 38, 249

Régulo, C., García, R.A., \& Ballot, J., 2016, A\&A, 589, A103

Reinhold, T., \& Gizon, L., 2015, A\&A, 583, A65

Reinhold, T., \& Reiners, A., 2013, A\&A, 557, A11

Reinhold, T., Reiners, A., \& Basri, G., 2013, A\&A, 560, A4

Salabert, D., García, R.A., Beck, P.G., et al., 2016a, A\&A, 596, A31

Salabert, D., García, R.A., Jiménez, A., et al., 2017, A\&A, 608, A87

Salabert, D., Régulo, C., Ballot, J., García, R.A., \& Mathur, S., 2011, A\&A, 530, A127

Salabert, D., Régulo, C., García, R.A., et al., 2016b, A\&A, 589, A118

Salabert, D., Régulo, C., Pérez Hernández, F., \& García, R.A., 2018, A\&A, 611, A84

Sandage, A., 1962, ApJ, 135, 349

Santos, A.R.G., Campante, T.L., Chaplin, W.J., et al., 2018, ArXiv e-prints

Santos, A.R.G., Cunha, M.S., Avelino, P.P., García, R.A., \& Mathur, S., 2017, A\&A, 599, A1

Schunker, H., Schou, J., Ball, W.H., Nielsen, M.B., \& Gizon, L., 2016, A\&A, 586, A79

Serenelli, A., Johnson, J., Huber, D., et al., 2017, ApJS, 233, 23

Sills, A., \& Pinsonneault, M.H., 2000, ApJ, 540, 489

Silva Aguirre, V., Lund, M.N., Antia, H.M., et al., 2017, ApJ, 835, 173

Skumanich, A., 1972, ApJ, 171, 565

Smith, J.C., Stumpe, M.C., Van Cleve, J.E., et al., 2012, PASP, 124, 1000

Stello, D., Cantiello, M., Fuller, J., et al., 2016, Nature, 529, 364

Stumpe, M.C., Smith, J.C., Catanzarite, J.H., et al., 2014, PASP, 126, 100

Stumpe, M.C., Smith, J.C., Van Cleve, J.E., et al., 2012, PASP, 124, 985

Tayar, J., \& Pinsonneault, M.H., 2013, ApJ, 775, L1

Thompson, M.J., Christensen-Dalsgaard, J., Miesch, M.S., \& Toomre, J., 2003, ARA\&A, 41,599

Thompson, M.J., Toomre, J., Anderson, E., et al., 1996, Science, 272, 1300

Tripathy, S.C., Kumar, B., Jain, K., \& Bhatnagar, A., 2001, Sol. Phys., 200, 3

van Saders, J.L., Ceillier, T., Metcalfe, T.S., et al., 2016, Nature, 529, 181

Vida, K., Oláh, K., \& Szabó, R., 2014, MNRAS, 441, 2744

Wilson, O.C., 1978, ApJ, 226, 379 